\title{
Extracellular nucleosides and nucleotides as
}

\section{immunomodulators}

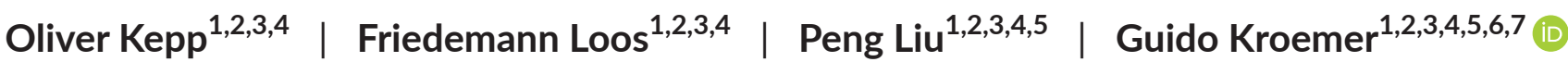

\author{
${ }^{1}$ Metabolomics and Cell Biology \\ Platforms, Gustave Roussy Comprehensive \\ Cancer Institute, Villejuif, France \\ ${ }^{2}$ Equipe 11 Labellisée Ligue Contre \\ le Cancer, Centre de Recherche des \\ Cordeliers, INSERM U 1138, Paris, France \\ ${ }^{3}$ Sorbonne Paris Cité, Université Paris \\ Descartes, Paris, France \\ ${ }^{4}$ Université Pierre et Marie Curie, Paris, France \\ ${ }^{5}$ University of Paris Sud XI, Kremlin Bicêtre, \\ France \\ ${ }^{6}$ Pôle de Biologie, Hôpital Européen Georges \\ Pompidou, AP-HP, Paris, France \\ ${ }^{7}$ Karolinska Institute, Department of Women's \\ and Children's Health, Karolinska University \\ Hospital, Stockholm, Sweden

\section{Correspondence} \\ Guido Kroemer, MD, PhD, Centre de \\ Recherche des Cordoliers, UMR1138 Équipe \\ 11, Paris, France. \\ Email: kroemer@orange.fr
}

\begin{abstract}
Summary
Some anticancer agents induce immunogenic cell death that is accompanied by the emission of danger signals into the tumor microenvironment, thus attracting and activating innate immune effectors and finally inducing anticancer immunity. The release of extracellular nucleosides such as adenosine triphosphate (ATP) from the tumor in response to anticancer therapy plays a pivotal role in the attraction of antigen presenting cells and the activation of inflammasome-mediated proinflammatory cascades. In contrast, the ectonucleotidase-catalyzed phosphohydrolysis of nucleotides to nucleosides reduces the extracellular availability of nucleotides, hence limiting the recruitment and activation of antigen-presenting cells. In addition, the (over-)production of nucleosides including adenosine by ectonucleotidases located on cancer cells and regulatory $T$ cells can induce immunosuppression, as adenosine directly inhibits the proliferation and activation of effector T cells. Here, we discuss the importance of death metabolites for immunomodulation in general, and the role of the purine nucleotide ATP and its derivative adenosine in particular. In addition, we provide an overview on therapeutic interventions that reinstate tumor immunogenicity in conditions where nucleotide-dependent immunostimulation is obstructed.
\end{abstract}

\section{KEYWORDS}

adaptive immunity, anticancer chemotherapy, cancer, immunogenic cell death

\section{1 | INTRODUCTION}

Every second, each individual cell in the healthy human body undergoes a multitude of energy-dependent biochemical reactions. Purine (and to a certain extent pyrimidine) nucleotides are universal transporters of chemical energy within cells. In particular, the adenosine nucleotide ATP serves as molecular currency for energy transfer. Summing up all energy-dependent processes in the healthy human body, the average daily molecular consumption of ATP is close to body weight, contrasting with the overall rather limited mass of adenosine nucleotide molecules (to roughly $50 \mathrm{~g}$ in an average adult). ${ }^{1}$ This discrepancy can be explained by the rapid recycling of ATP and points to the limited availability of this metabolic key molecule for mechanisms other than energy transfer. It also underscores its strictly intracellular localization under homeostatic conditions, as both consumption as well as recycling occur inside of the cell and diminishing the intracellular pool of ATP would lead to an energetic crisis. ATP is stored intracellularly at close to micromolar concentration, but is rapidly degraded upon its release by extracellular hydrolases. This hydrolytic degradation further limits the extracellular ATP concentration and establishes a steep concentration gradient over the plasma membrane. ${ }^{2}$ Thus, nucleotides in general and ATP in particular exhibit optimal prerequisites for serving as extracellular signaling molecules, and their release from even a few cells only causes a significant increase in their extracellular abundance. Besides its well-established role in purinergic neurotransmission, ATP and its derivatives serve as signaling molecules in various cellular processes including vasodilation, mucociliary clearance, and immunomodulation. ${ }^{3-6}$ Intracellular nucleotides can be released in response to a variety of cell stress- and cell death-associated conditions including shear stress following physical distortion, plasma membrane 
damage, hypoxia or stress elicited by cytotoxic agents including anticancer chemotherapeutics. ${ }^{3-6}$

The release of intracellular molecules can serve as adjuvant, stimulatory signals for sensors of the innate and adaptive immune systems. In response to cellular stress and death, metabolites such as nucleotides are released from the cell into the extracellular space, where ectonucleotidases can hydrolyze and convert them into nucleosides. Both, nucleotides or nucleosides play a pivotal role in shaping the immune response to dying cells. Thus, nucleotide-mediated ligation of purinergic receptors of the P2R family on antigen presenting cells (APCs) drives their chemotactic recruitment for the stimulation of an immune response. In contrast nucleoside binding to $\mathrm{P} 1 \mathrm{R}$ receptors on effector T cells has immunosuppressive effects. ${ }^{7,8}$

Several chemotherapeutic anticancer agents have been identified as being superior inducers of anticancer immune responses which improve long-term therapeutic efficacy mediated by the generation of immunological memory. Immunogenic cell death (ICD) strongly depends on the release of ATP from dying cells as well as on its stability in the extracellular space of the tumor. ${ }^{9}$ Thus, the autophagy-dependent release of the nucleotide ATP via lysosomal exocytosis during ICD and its binding to $\mathrm{P} 2 \mathrm{RX} 7$ on dendritic cells (DCs) is decisive for the generation of adaptive immunity against tumor-derived antigens. ${ }^{10}$ Several different ectonucleotidases expressed on the surface of tumor or regulatory $T$ cells $\left(T_{R E G}\right)$ can catalyze the phosphohydrolysis of extracellular ATP to adenosine. ${ }^{11}$ Adenosine inhibits effector T-lymphocyte proliferation and the secretion of inflammatory cytokines, and is therefore critical for both innate and adaptive immune responses. ${ }^{12}$ Recently, two different ectonucleotidases, namely ectonucleoside triphosphatediphosphohydrolase 1 (ENTPD1, better known as CD39) and ecto- $5^{\prime}$-nucleotidase (NT5E, better known as CD73), as well as $A 2 A R$ and $A 2 A B$, the P1R adenoside receptors on effector T cells and DCs, respectively, have emerged as targets for anticancer immunotherapeutic approaches. ${ }^{13,14}$ Here, we elaborate the idea that a combination of anticancer regimens that, on one hand, facilitates the release of nucleotides and, on the other hand, limits their degradation to nucleosides would have high prospects of clinical success.

\section{2 | CELL DEATH MODALITIES AND IMMUNITY}

Every second millions of cells undergo cell death in the healthy human body. This demise is immunologically silent as it is part of the homeostatic turnover that safeguards tissue integrity. Dying cells are removed by professional phagocytes such as macrophages and efferocytotic neighboring cells without eliciting a detrimental inflammatory response against dead cell-associated antigens. ${ }^{15}$ Only in pathological conditions, this evolutionary highly conserved mechanism derails and potentially culminates in fatal autoimmune reactions. For long the prevailing scientific opinion favored a dichotomy that classified apoptotic and necrotic instances of cell death into physiological and proinflammatory cell death events, respectively. Apoptosis was viewed as regulated cell death (RCD) whereas necrosis was considered as pathological, accidental cellular demise. ${ }^{16}$ During recent years, this oversimplified model has been challenged at multiple levels. First the notion that all forms of RCD remain immunologically silent or even confer immunotolerance has been confronted with the fact that certain physical or chemical treatments, including anthracyclin- or oxaliplatin-based chemotherapies, treatment with cardiac glycosides or certain cytolytic compounds, UV- or $\gamma$-irradiation, hypericin-based photodynamic therapy (PDT), and high hydrostatic pressure (HHP), have the capacity to induce traits of ICD and to convert dying tumor cells into therapeutic vaccines. ${ }^{17-22}$ Thus, MCA205 fibrosarcoma, TC1 lung cancer or EL4 lymphoma cells succumbing to ICD have the ability to immunize immunocompetent syngenic C57/BL6 mice against a subsequent rechallenge with isogenic tumor cells of the same type. ${ }^{23-}$ ${ }^{25}$ Similar effects were achieved in other syngenic systems including CT26 colon carcinoma inoculated into BALB/c mice ${ }^{19}$; Second, regulated forms of necroptosis were discovered that depend on the receptor-interacting serine/threonine kinase 3 (RIPK3)-mediated phosphorylation of the pseudokinase mixed lineage kinase domainlike (MLKL), its subsequent oligomerization and finally membrane permeabilization. Despite being yet another form of RCD, accumulating evidence suggests that necroptosis-deficient tumors are less immunogenic than their necroptosis-proficient counterparts, which might (in part) explain why necroptosis-deficient cells can escape natural and therapy-elicited immunosurveillance. This goes in line with recent reports describing necroptotic forms of ICD. The combined treatment of TC1 lung cancer or EL4 lymphoma cells with tumor necrosis factor alpha (TNF $\alpha$ ), the pan-caspase inhibitor Z-VAD-fmk and an inhibitor of apoptosis proteins (IAP)-binding second mitochondria-derived activator of caspases (SMAC) mimetic triggered several hallmarks of ICD. CRISPR-mediated knockout of Rip3k or Mlkl abolished the emission of immunogenic factors such as the release of ATP. ${ }^{26}$ In addition, TC1 cells treated with the ICD inducer mitoxantrone exhibited signs of necroptosis, and mitoxantrone was less efficient in conferring protective immunity in vivo when the tumor cells used for vaccination were deficient in either Ripk3 or Mlkl. Restoring immunogenic signaling by means of compensating for deficient ATP and high mobility group box one (HMGB1) release restored the efficacy of chemotherapy in $\mathrm{Rip3}^{-/-}$and $\mathrm{Mlkl}^{-/-}$cancers. ${ }^{26}$

\section{3 | CELL DEATH AND DANGER SIGNALING}

The mammalian innate immune system provides a first line of defense against pathogenic infection that relies on the detection of pathogenassociated molecular patterns (PAMP) including bacterial lipopolysaccharide (LPS), flagellin, and peptidoglycans as well as viral double stranded RNA (dsRNA). PAMP are sensed by specialized pattern recognition receptors (PRR) on a variety of immune cells including macrophages, monocytes, DCs and additional components of the innate immune system. ${ }^{27}$ The spectrum of molecules sensed by PRR is not limited to exogenous PAMP but includes host-derived endogenous molecules which are released in response to certain kinds of cellular stress and death. ${ }^{28}$ In this scenario, specific damage-associated 
molecular patterns (DAMP) released by stressed and dying cells during the course of ICD are sensed by PRR and can thus act as adjuvants that by themselves initiate innate immune response. ${ }^{29,30}$ Among the known features of ICD, the extracellular death metabolite ATP plays a pivotal role. In the course of cell death ATP is released from dying cells and ligates purinergic receptors of the P2RX7 and P2RY2 type to drive the chemotactic recruitment of $\mathrm{APC}$ as well as their activation, respectively. In the course of ICD, the autophagy-dependent ATP secretion and the interaction of the metabolite with $\mathrm{P} 2 \mathrm{RX} 7$ drives the recruitment and activation of DC.

Other ICD-specific hallmarks include first, the exposure of calreticulin (CALR) on the cell surface in the initiation phase of ICD. CALR serves as de novo uptake signal for low-density lipoprotein receptorrelated protein 1 (LRP1) expressed on DC, thereby promoting the engulfment of dead cell-associated antigens. ${ }^{19,20}$ Second, Annexin A1 (ANXA1) is released from dying cells in the course of ICD and binds to formyl peptide receptor 1 (FPR1) expressed on DC and thus serves as an additional chemotactic signal to direct the final approach of APC towards dying cancer cells. ${ }^{31}$ Third, the release of CXCL10 from the tumor as a consequence of autocrine or paracrine type I interferon (IFN) signaling and its ligation of CXCR3 stimulates the supplementary recruitment of T cells. ${ }^{32}$ Fourth, HMGB1, that is passively released during final stages of cell death, ligates several receptors including advanced glycosylation end-product specific receptor (AGER), Toll-like receptor (TLR) 2 and TLR4, and triggers immunostimulatory responses including synthesis of the pro-inflammatory type I IFN. Its role in ICD depends on the TLR4 MyD88 signaling route and the subsequent release of interleukin (IL)-1 $\beta$ by activated DC (Figure 1). ${ }^{33}$ However, neither PAMP nor DAMP are able to trigger adaptive immunity alone unless antigenic neo-epitopes from either microbial or oncogenic origin, which have not provoked central or peripheral tolerance earlier, are present. $^{34}$

In summary, adaptive immune responses to ICD depend on a combination of neo-epitope-mediated antigenicity together with increased adjuvanticity conferred by the presence of DAMP including the immunogenic death metabolite ATP. In an optimal combination, therapy-induced ICD will thus yield immunological memory against tumor antigens and confer relapse-free survival.

\section{4 | NUCLEOTIDE RELEASE MECHANISMS}

All cells in the human body dispose of mechanisms for the release of ATP from the cellular lumen to the extracellular environment, underlining the importance of ATP as a molecular messenger. ${ }^{35}$ When cells undergo apoptosis in response to cytotoxic stimuli including chemotherapeutic agents, cells release nucleotides into the extracellular space to promote the chemotaxis of phagocytes, dead cell engulfment, and clearance. ${ }^{36,37}$ Cell stress and cell death-induced release of ATP can depend on a variety of mechanisms such as the exocytosis of vesicular ATP, ${ }^{38}$ the connexin-mediated secretion of cytoplasmic ATP via gap junction hemi-channels, ${ }^{39}$ the secretion via pannexin channels ${ }^{40}$ or trans-membrane transport via ATP-transporters specific for the ATP-binding cassette family such as the cystic fibrosis transmembrane conductance regulator. ${ }^{41}$

An exocytotic release of ATP in analogy to its liberation from sympathetic perivascular nerve terminals has initially been proposed in the context of ICD. Indeed, ATP appears to be enriched in intracellular vesicles in cells labeled with the quinolone-acridine derivative quinacrine, that exhibits a high binding affinity for ATP. ${ }^{42,43}$ Meanwhile it has become clear that the release of ATP from intracellular vesicles is a common phenomenon in a large variety of tissues not only limited to secretory cells. ${ }^{44}$ Exocytotic vesicles arise from the Golgi apparatus, where cellular content originating from the cytosol or the endoplasmic reticulum (ER) is packaged. The vesicles traffic toward the cytoplasmic membrane and their content is released into the extracellular space upon membrane fusion. ${ }^{45}$ Recently, the mechanism sustaining vesicular ATP concentration was reported to involve the vesicular nucleotide transporter solute carrier 17A9 (SLC17A9) that depends on the proton gradient maintained by the vacuolar-ATPase (v-ATPase). Cellular machineries that are involved in alternative ATP release mechanisms include certain members of the group of $A B C$ transporter, such as the cystic fibrosis transmembrane conductance regulator (CFTR), ${ }^{46}$ ATP binding cassette subfamily $\mathrm{C}$ member 8 (ABCC8) better known as sulfonylurea receptor (SUR), ${ }^{47}$ and the ATP binding cassette subfamily $B$ member 1 (ABCB1) also known as the multidrug resistance gene product MDR. ${ }^{48}$ All these transporters have been shown to transport purine nucleotides in an energy-dependent process to the
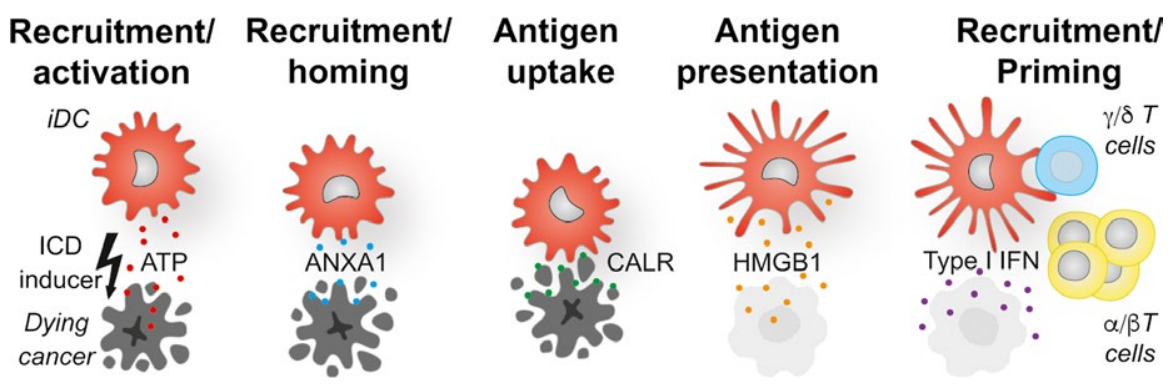

FIGURE 1 Chemotherapy-induced purinergic immune stimulation. Immunogenic cell death (ICD)-inducing anticancer chemotherapy causes the release of ATP from tumor cells into their microenvironment. The mechanism of release comprises (part of) the autophagic machinery as well as caspase-3 (CASP-3)- and pannexin 1 (PANX1)-dependent reactions and hence can be inhibited at multiple levels (with inhibitors of autophagy, CASP-3 and PANX1 channel activity). The immunostimulatory functions of ATP include the attraction of antigen presenting cells into the tumor bed and the activation of NLRP3 inflammasomes in dendritic cells (DC) that finally result in the release of interleukine (IL)-1 $\beta$ and the activation of cytotoxic T lymphocytes (CTL) 
extracellular space for autocrine/paracrine purinergic signaling. ${ }^{46,48}$ In certain cell types, F1Fo ATPase complexes are ectopically localized at the plasma membrane with the catalytic F1 head facing outwards. Reportedly, this complex can synthesize ATP from ADP and inorganic phosphate directly in the extracellular space. Ectopic F1Fo ATPase expression in vascular endothelial cells underpins its role in regulating vascular purinergic signaling events. ${ }^{49}$ Yet another mechanism for ATP release was described for connexins that are gap junction proteins localized at the plasma membrane. In close apposition with adjacent cells, they are able to form gap junction channels and allow for cellto-cell communication. In pathological conditions, including inflammatory responses, connexin hemichannels at the surface of several cell types can function as conduits for the transport of ATP molecules into the extracellular space. ${ }^{39,50}$ Similar to connexins, the members of the pannexin family, with thus far three identified isoforms, form voltage gated channels in the plasma membrane that are permeable to ATP. The caspase-dependent proteolytic activation of pannexin 1 (PANX1) in apoptotic cells mediates ATP release into the extracellular milieu and the subsequent recruitment of phagocytes. ${ }^{36}$ RNA interference-mediated silencing of PANX1 expression leads to a significant reduction in ATP release, as well as mitigated monocyte/leucocyte recruitment towards anti-FAS treated or UV irradiated cells. In accordance with these findings, thymocytes from Panx $\mathrm{P}^{-/-}$mice treated with dexamethasone displayed impaired ATP release and were unable to promote macrophage chemotaxis. ${ }^{51}$

ICD-related release of ATP is a multistep process that involves lysosomal exocytosis of the metabolite into the pericellular space. Thus, when ICD is triggered, intracellular ATP molecules relocate from the lysosomal compartment to the lumen of autolysosomes and are subsequently secreted by the molecular exocytosis machinery that involves the lysosomal protein lysosomal associated membrane protein 1 (LAMP1) and its PANX1-dependent translocation. The redistribution of LAMP1 to the cytoplasmic membrane, the activation of Rho-associated, coiled-coil containing protein kinase 1 (ROCK1)-mediated cellular blebbing, as well as the gating of pannexin 1 (PANX1) channels occurs in a strictly caspase-dependent manner. Thus, lysosomal exocytosis plays a major role in ICD-induced caspaseand PANX1-dependent ATP secretion ${ }^{52-54}$ (Figure 2). Despite these findings, the detailed mechanism of ICD-induced ATP release remains partially elusive. In addition, ATP secretion might be restricted to a very limited timeframe during cell death as it has been shown that during apoptosis induced by staurosporin or TNF-related apoptosisinducing ligand (TRAIL), the level of intracellular ATP decreases due to the caspase-dependent inhibition of the glycolytic enzymes phosphofructokinase and pyruvate kinase. ${ }^{55}$

\section{5 | AUTOPHAGY IN PURINERGIC SIGNALING}

Macroautophagy (from here on called autophagy) is a cellular stress response process that involves the sequestration of cytoplasmic content into double-membraned autophagosomes. In the course of autophagy, lysosomal fusion leads to the degradation of autophagosomal content by lysosomal hydrolases. Thus, autophagic recycling serves as an important source of substrates for energy-dependent metabolic or anabolic reactions. ${ }^{56}$ The soluble $\mathrm{N}$-ethylmaleimide-sensitive factor attachment protein receptor (SNARE) protein vesicle associated membrane protein 7 (VAMP7) has been associated with ATP-release from autophagosomes. ${ }^{57}$ The inhibition of autophagy by genetic or pharmacological intervention at the level of several essential regulators including autophagy related (ATG)5, ATG7, Beclin 1(BECN1), LAMP2, phosphatidylinositol 3-kinase VPS34 (VPS34) abolishes the secretion of ATP in cells that succumb to ICD or viral infection. In addition, it suppresses their immunogenicity. ${ }^{58,59}$ Anthracycline-based chemotherapy induces cell death independent of autophagy, but autophagy
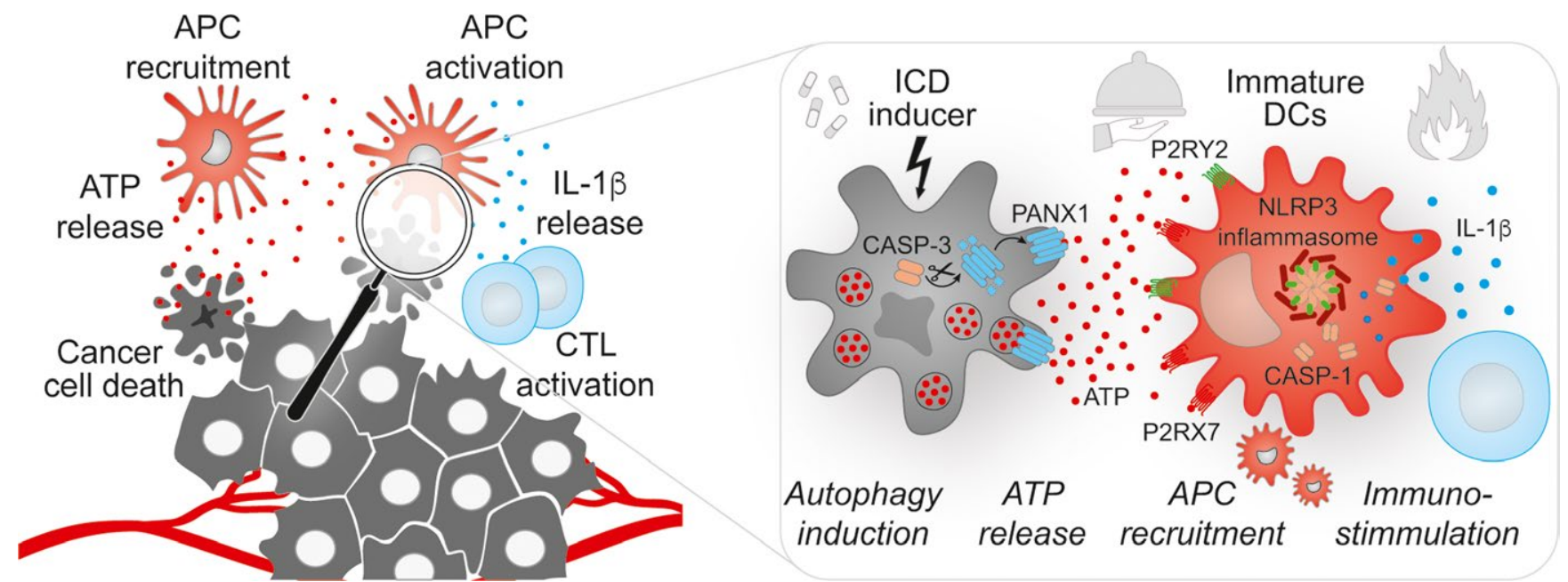

FIGURE 2 Release or exposure of DAMPs in response to immunogenic cell death. Together with annexin A1 (ANXA1), that guides immune cells towards dying cells, ATP serves as chemoattractant and as stimulatory factor for immature dendritic cells (DC). Calreticulin (CALR) acts as de novo uptake signal for DC on dying cells, while high mobility group box 1 (HMGB1) leaks out of dead cells and then supports together with Type I interferons (IFN) the maturation of DC as well as tumor antigen presentation by DC to T lymphocytes 
is required for chemotherapy-induced immunogenicity as it facilitates the immunostimulatory release of ATP over the immunosuppressive generation of adenosine. ${ }^{60}$ Thus, autophagy-deficient tumors in mice exhibit an impaired recruitment of monocytes, macrophages, and DC into the tumor microenvironment, lack of antitumor immune responses, and they do not respond to therapeutic interventions. In this context, the local injection of ectonucleotidase inhibitors led to an increase in the extracellular ATP content in autophagy deficient tumors, thereby re-establishing the recruitment of immune effectors into the tumor bed. ${ }^{58}$ Such intervention also re-established the immune cell recruitment and restored chemotherapeutic responses in immunocompetent animals. Thus, autophagy induction by chemotherapy is mandatory for the immunogenic secretion of ATP from stressed and dying tumor cells, and ultimately necessary to elicit anticancer immunity. ${ }^{58}$ Of note, although autophagy and LAMP1 fail to influence PANX1 channel opening, PANX1 is required for the ICD-associated translocation of LAMP1 to the plasma membrane. ${ }^{52}$ Recently, it was reported that interventions inducing systemic autophagy, such as fasting or the administration of caloric restriction mimetics, improved the therapeutic outcome of immunogenic chemotherapy, and correlated with the depletion of $\mathrm{T}_{\text {REG }}$ from the TME. ${ }^{61}$ In summary, the autophagic machinery safeguards sufficient energy resources during cellular stress conditions and maintains an elevated availability of intracellular nucleotides. In addition, part of the molecular machinery driving autophagy is involved in ATP secretion from stressed and dying cells, explaining the link between autophagy occurring in malignant cells and anticancer immune responses.

\section{6 | NUCLEOTIDE PHOSPHOHYDROLYSIS AND IMMUNITY}

Local purinergic signaling changes over time from an acute phase of ATP release and a high ratio of ATP/adenosine to a subacute phase with reduced ATP release by the induction of ectonucleotidases that change decrease the nucleotide/nucleoside ratio. ${ }^{35}$ Ectonucleotidases are membrane-anchored nucleotide phosphohydrolyzing proteins that under homeostatic conditions serve to minimize the content of extracellular nucleotides. The conversion of extracellular nucleotides to nucleosides has been best studied in the case of ATP, which yields adenosine. This conversion results from a multistep process that requires several enzyme-catalyzed reactions. Ectonucleoside triphosphate diphosphohydrolase 1 (ENTPD1 better known as CD39) hydrolyzes ATP to ADP and further to AMP. This step can be antagonized by extracellular kinases such as adenylate kinase, which reverts the reaction. Extracellular AMP can be further converted into the immunosuppressive metabolite adenosine by ecto- 5 '-nucleotidase (NT5E, better known as CD73). Several studies have shown that the expression of CD39 on tumor cells, such as leukemia, ${ }^{62}$ gastric cancer, ${ }^{63}$ glioma, ${ }^{64}$ endometrial cancer, ${ }^{65}$ and colorectal cancer, ${ }^{66}$ but not pancreatic cancer, ${ }^{67}$ has a negative prognostic impact. In addition, CD39 is expressed in a variety of different non-carcinogenic cell types including hematopoietic and non-hematopoietic cells such as endothelial cells, B cells, NK cells, certain T cell subsets, macrophages and DCs. ${ }^{68,69}$ The expression of CD39 on tumor-infiltrating leukocytes, including $T_{\text {REG }}$ and a subset of Th17 cells, mediates (part of) their immunosuppressive effect and drives immune tolerance and tumor progression. ${ }^{70}$ Consistently, CD39 expression on $\mathrm{T}_{\text {REG }}$ promotes hepatic metastasis in a murine melanoma cancer model via the inhibition of NK cells. ${ }^{71}$ Tumor cells with disabled autophagy exhibit overexpression of CD39 and an increased attraction of $\mathrm{T}_{\text {REG }}$ into the tumor bed. ${ }^{72}$ In addition, transgene-enforced overexpression of CD39 on cancer cells subverts anticancer immune responses induced by ICD inducers in vivo, in models of chemotherapy, thus reducing therapeutic efficacy. ${ }^{58}$ Therefore, CD39 claims a prominent role in immunosuppression as a key protein for the limitation of extracellular ATP and as a source of substrates for the CD73-mediated hydrolysis of AMP to immunosuppressive adenine (Figure 3 ).

CD73 has a wide spectrum of substrates as it catalyzes the phosphohydrolysis of both purine and pyrimidine ribo- and deoxyribonucleoside monophosphates such as AMP to adenosine. Thus, CD73 mediates the rate-limiting reaction leading to the generation

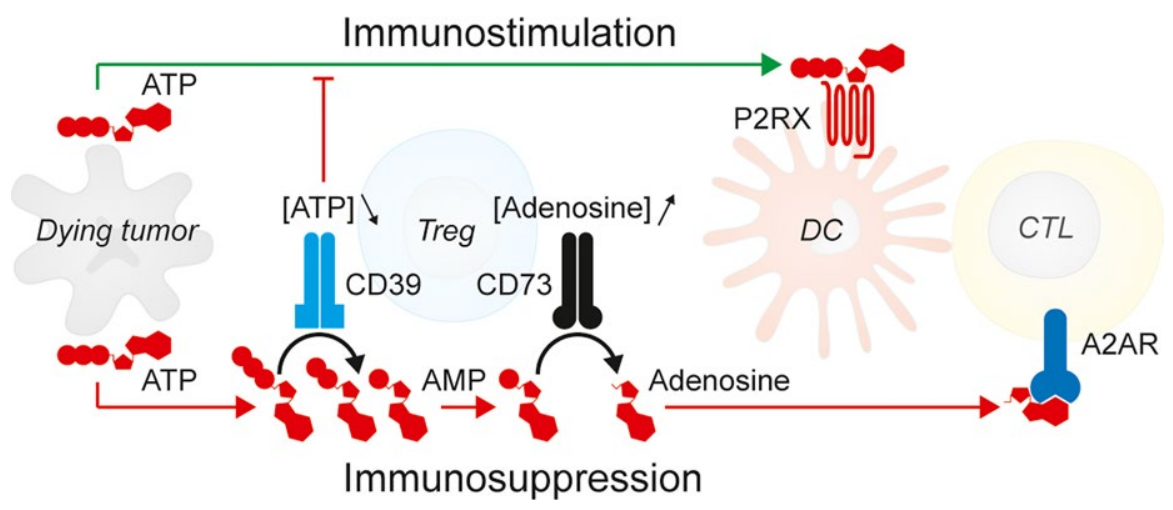

FIGURE 3 Ectonucleotidase-triggered immunosuppression. Extracellular release of ATP from dying tumor cells ligates receptors of the P2RXtype on dendritic cells (DC). The expression of both CD39 and CD73 ectonucleotidases on tumor cells and in particular on regulatory T cells $\left(\mathrm{T}_{\mathrm{REG}}\right)$ promotes the accumulation of ATP-derived adenosine in the tumor microenvironment and the resulting activation of immunosuppressive A2AR on cytotoxic T lymphocytes (CTL). Pharmacological interventions to reactivate immunosurveillance have been developed to block the production of adenosine or its action on A2AR receptors 
of extracellular adenosine. ${ }^{73}$ CD73 is expressed on a variety of cells, including cancers, and is associated with poor prognosis in breast cancer, ${ }^{74}$ ovarian cancer, ${ }^{75}$ prostate cancer, ${ }^{76}$ brain cancers, ${ }^{77}$ and leukemia. ${ }^{78}$ CD73 is also expressed on non-malignant cells such as $T_{\text {REG }}$, Th17 cells, myeloid-derived suppressor cells (MDSCs), B cells, endothelial and epithelial cells, as well as DCs. ${ }^{69,79}$ In cancerous cells and the tumor microenvironment, the expression level of CD73 can be directly regulated by hypoxia. The master transcriptional regulator that is activated in response to limited oxygen supply, hypoxia-inducible factor 1-alpha (HIF1A), binds to response elements located within the CD73 promoter and activates its expression. ${ }^{80,81}$ Other factors present in the tumor microenvironment including IL-1 $\beta$, IL-6, prostaglandin E2 (PGE2) and tumor growth factor beta (TGF- $\beta$ ) have also been associated with increased ectonucleotidase expression, whereas IFN- $\gamma$ and IL-4 inhibit CD73 expression. ${ }^{69,82}$

Mice deficient in the $\mathrm{Nt5e}$ gene coding for CD73 are protected against the development of spontaneous tumors such as genetically induced prostate cancers (in TRAMP mice) and chemically induced fibrosarcomas, due to the lack of adenosine-dependent immunosuppressive signaling and the resulting improved immunosurveillance. ${ }^{83}$ The impact of CD73 on anticancer immune responses is further underlined by the fact that ectopic expression of the ectonucleotidase from a plasmid reduces the efficacy of anticancer immunotherapy. ${ }^{84}$ Accordingly, monoclonal antibodies targeting CD73 are able to suppress the growth of chemically and genetically induced spontaneous tumors. ${ }^{84}$ This effect was suppressed by depletion of CD8 ${ }^{+}$T lymphocytes, underscoring that it relies on a cellular immune response. ${ }^{84}$

In summary, the secretion of ATP by stressed and dying tumor cells appears indispensable for anticancer immune responses. The expression of ectonucleotidases by cancer cells or tumor-infiltrating immunosuppressive cells can subvert ATP signaling toward an adenosine-dependent immunosuppression. It is thus likely that immunoediting selects cancer cells that exhibit an increased expression level of ectonucleotidases.

\section{7 | IMMUNE PERCEPTION OF NUCLEOTIDES AND NUCLEOSIDES}

The scientific view of the role of nucleotides and nucleosides as extracellular autocrine and/or paracrine signaling molecules has evolved over the past century and extensive work has been conducted to characterize purinergic receptors expressed on a plethora of different cell types. ${ }^{85}$ By now it is well established that all immune cells express receptors for extracellular nucleosides and nucleotides such as adenosine and ATP, respectively. ATP ligates all subtypes of the metabotropic P2YR and ionotropic P2XR purinergic receptor family with the only exception of $P 2 Y 12 R,{ }^{86}$ whereas pyrimidine nucleotides display a selective affinity for certain subtypes of the P2YR family including P2Y2R, P2Y4R, P2Y6R and P2Y14R. ${ }^{64}$ An oncogenic role of $P 2 R X 7$ has been ascribed to its interaction with the VEGF pathway as direct interactor of VEGFR2 and a potent stimulator of VEGF release. ${ }^{87,88}$
Depending on the specific receptor, extracellular purines can initialize immunosuppressive or immunostimulatory responses. Recently, the nucleotide-derived metabolites adenine and N4-acetylcytidine have been detected in the blood of patients with low-grade chronic inflammation. ${ }^{67}$ Both adenine and $\mathrm{N} 4$-acetylcytidine can induce priming and activation of the NLRC4 inflammasome, which in turn induces interleukin-1 $\beta$ (IL-1 $\beta$ ) production. Moreover, adenine and N4acetylcytidine injected into mice can cause hypertension and arterial stiffness. ${ }^{89}$ Interestingly, ATP is also released from neutrophils in response to FPR1 stimulation. The pharmacological inhibition or siRNAmediated silencing of pannexin hemichannels or P2Y2 receptors blocked the activation of neutrophils and abrogated innate immune responses to bacterial infection, underlining the importance of ATP purinergic signaling for the immune defense. ${ }^{45}$

Extracellular nucleotides comprising ATP and UTP have also been identified as essential apoptotic find-me signals. ${ }^{7}$ Thus, caspasedependent generation of a steep chemotactic gradient around dying cells promotes the recruitment of myeloid cells via ligation of P2RY2 on their surface, an effect that was recapitulated by adding purified nucleotides and abrogated by apyrase-mediated enzymatic removal of purines. ${ }^{7,36}$ Infiltration of monocytes and macrophages induced by apoptotic supernatants was reduced when nucleotides were depleted in vivo or when the recruitment was monitored in $\mathrm{P} 2 \mathrm{ry} 2^{-/-}$animals. Similarly, both interfering with $\mathrm{P} 2 \mathrm{Y}$ receptor function and depletion of nucleotides prevented adequate removal of apoptotic thymocytes. ${ }^{7}$ In line with these findings, ICD-mediated immune responses are suppressed when the tumor microenvironment lacks cell death-derived nucleotides or when purinergic signaling via P2RX7 is affected. ${ }^{10,24,58}$ Purinergic signaling promotes the activation of the NLR family, pyrin domain containing 3 (NIrp3) inflammasome in murine DC, caspase-1 activation and the processing and immunostimulatory release of IL$1 \beta$. This release results in activation of IL-17-producing $\gamma \delta \mathrm{T}$ cells that precedes the accumulation of IFN- $\gamma$-producing $\mathrm{CD}^{+} \alpha \beta$ T lymphocytes within the tumor bed. ${ }^{10,90}$ Accordingly, $\mathrm{P} 2 \mathrm{rx}^{-/-}, \mathrm{Nlrp3}^{-/-}$, Casp-1 $1^{-/-}, 1117 a^{-/-}$, or $1117 \mathrm{ra}^{-/-}$mice, as well as animals treated with an IL-1 $\beta$-neutralizing antibody lack the ability to mount adaptive immune responses against cancer cells undergoing ICD. ${ }^{10,90}$ In a cohort of patients with breast carcinoma, a loss-of-function polymorphism in P2RX7, which lowers its affinity for ATP, negatively affected disease outcome after anthracycline-based chemotherapy. ${ }^{10}$ Conversely, the expression of P2RX7 on cancer cells has been associated with negative prognosis in several types of malignancies including acute myeloid leukemia, prostate cancer, and neuroblastoma. ${ }^{91}$ Similar to ATP, ADP fulfills immunostimulatory functions, such as the P2RY12-mediated stimulation of antigen uptake by splenic DC. ${ }^{92}$

Adenosine produced by ectonucleotidases that are expressed on either the tumor or immunosuppressive cells that infiltrate the tumor microenvironment such as $\mathrm{T}_{\mathrm{REG}}$, Th17 and MDSCs, binds to purinergic A2A receptors on CTLs and NK cells and inhibits the immune reaction against cancer cells. Ligation of the G-protein-coupled A2AR (as well as to a certain extent of $A 2 B R$ ) on immune effectors triggers the intracellular accumulation of cyclic AMP (c-AMP), which in turn binds to the regulatory subunit of c-AMP-dependent PKA. The resulting cascade 
of phosphorylation events inhibits $T$ cell receptor (TCR)-dependent proinflammatory signaling necessary for efficient CTL activation, expansion and IFN- $\gamma$ production. ${ }^{93}$ Thus, adenosine disables cytotoxic anti-tumor immunity, while fostering several adverse effects such as tumor growth-supporting neovascularization and de novo infiltration of immunosuppressive cells. ${ }^{94}$ Modulating adenosine levels in the TME is a promising approach to limit tumor progression and reinstate tumor immunosurveillance.

\section{8 | THERAPEUTIC MODULATION OF PURINERGIC SIGNALING}

Mouse lymphoma EL4 or EG7 tumors transplanted on P2xr7-deficient mice failed to mount a tumor-specific $\mathrm{CD}^{+} \mathrm{T}$ cell response when treated with immunogenic chemotherapeutic agents such as oxaliplatin. ${ }^{10}$ This phenotype was reverted when wildtype DC were adoptively transferred, ${ }^{10}$ indicating that ATP-dependent activation of the NLRP3 inflammasome plays a crucial role for therapeutic efficacy.

Pharmacological intervention specifically designed to enhance immunogenicity of cancer cells can target at least three different scenarios. First, ATP that is released in response to ICD-inducing agents can be stabilized in tumors that overexpress CD39 and CD73 ectonucleotidases to allow the onset of an immune response and to minimize the therapy-opposing action of adenosine on tumor suppressor cells. Thus, the use of chemical ectonucleotidase inhibitors or monoclonal antibodies that can block their function may be expected to increase the immunogenicity of anticancer treatments. Second, ATP-release in response to anticancer agents that by themselves do not yield sufficient release of the death metabolite may be amplified by stimulating the ATP secretion machinery of the tumor. So far, the sole ATP secretagogues are autophagy inducers, ${ }^{61}$ although it is tempting to speculate that other ATP releasing agents might be developed in the near future. Third, tumors that lack (parts of) the ATP releasing machinery might be treated by local injection of ATP agonists.

CD39, expressed on certain cancers and $\mathrm{T}_{\mathrm{REG}}$, can be targeted by monoclonal antibodies as well as pharmacological apyrase inhibitors such as the small-molecular inhibitor polyoxometalate-1 (POM-1) in order to increase the concentration of ATP into TME and to limit the production of immunosuppressive adenosine. Nevertheless, due to probable side effects, none of the strategies of CD39 targeting has been tested in cancer patients.

Recent evidence supports the notion that the CD73-catalyzed phosphohydrolysis of AMP to adenosine can be defined as a novel immune checkpoint (or at least a complementary target) that plays a decisive role in tumor immune escape. In clinical practice, combination therapies employing antagonists of multiple immunosuppressive mechanisms might be needed to overcome the numerous redundant pathways that elicit immunosuppression. In line with this idea, a clinical trial (NCT02503774) is currently evaluating the efficacy of anti-CD73 antibody (MEDI9447) alone or in combination with antiPD-L1 (MEDI4736) in solid malignancies. The small-molecular compound adenosine $5^{\prime}$-( $\alpha, \beta$-methylene)diphosphate (APCP) specifically inhibits the catalytic function of CD39, and has been shown to inhibit tumor growth and metastasis in several preclinical models. ${ }^{71,95}$ Thus, pharmacological inhibitors of CD73 might reach the clinics as well.

Adenosine effects may be neutralized by antibodies or small molecules that target the purinergic adenosine A2A receptors expressed on CTLs and NK cells. Promising work showed that A2AR-deficient mice rejected immunogenic melanomas. ${ }^{96}$ Moreover, A2AR antagonists such as caffeine or the RNAi-mediated silencing of A2AR on T cells improved the inhibition of tumor growth. ${ }^{96}$ These effects were attributed to a direct action on $\mathrm{CD}^{+}{ }^{+} \mathrm{TCL}$ or the de-inhibition of several DC subsets resulting in improved antigen presentation and cytokine mediated T cell activation. ${ }^{97}$ Antagonists of A2AR such as $\mathrm{SCH} 420814$ from Merck entered into clinical testing for Parkinson's disease, yet have not been employed for immuno-oncology. ${ }^{98,99}$ Of note, in nonmalignant tissues, immunosuppressive adenosine serves to protect cells from overzealous immune responses following pathogen infection and inflammation. ${ }^{100}$ Recently, autoimmune reactions have been detected in A2AR-deficient mice during the rejection of transplanted melanoma, indicating that the adenosinergic pathway in $\mathrm{T}$ cells plays an important role in maintaining autotolerance. Thus, pharmacological inhibitors or antibodies that reduce the adenosinergic signaling may be expected to cause autoimmune side effects. ${ }^{96}$

\section{9 | CONCLUSION}

Anticancer chemotherapies have been conceived to eradicate highly proliferative cells including cancer cells and-as a side effect-hematopoietic and immune cells. Immunosuppression is therefore a common side effect of clinically employed anticancer agents and potentially results in therapeutic failure. Many anticancer regimens used in clinical routine thus far lack long-term therapeutic success, meaning that patients relapse due to the emergence of therapy-resistant tumor clones. ICD has emerged as a strategy for converting cancer cells into a therapeutic vaccine. The release of immunogenic DAMP including ATP from dying cancer cells as well as the perception of such DAMP by the host's immune system constitute the prerequisites of therapeutically relevant ICD. Immunogenic anticancer chemotherapies have been proven to be superior in reducing the growth of cancer cells in immunocompetent animals compared to agents that fail to induce ICD. ${ }^{19,22,101,102}$ Recently, immune checkpoint blockers comprising antibodies targeting PD-1, PD-L1, and CTLA-4 have entered the clinical arena. PD-1/PDL-1 blockade was demonstrated to be extraordinarily effective in certain highly immunogenic cancers such as melanoma ${ }^{103}$ and even non-small cell lung cancer. ${ }^{104}$ Nevertheless, the efficacy of cancer immunotherapies depends on the cancer immune set point that can be defined as the equilibrium between factors that support or suppress anticancer immunity. ${ }^{105,106}$ ICD-inducing chemotherapies can stimulate the infiltration of cancers by $\mathrm{CD} 8^{+}$cytotoxic $\mathrm{T}$ lymphocytes. ${ }^{101}$ The enrichment of the TME with infiltrating T cells also sensitizes cancers to checkpoint inhibition, as this has been shown for KRAS proto-oncogene GTPase-induced lung cancers in mice treated first with chemotherapy (oxaliplatin + cyclophosphamide) and then 
with checkpoint blockade (anti-CTLA-4 + anti-PD1). ${ }^{107}$ As a consequence, the proportion of cancers that respond to immune checkpoint blockade might be substantially expanded by a combination therapies that involve ICD inducers together with checkpoint blockers. ${ }^{107}$

During tumor development, inefficient ATP release from cancer cells or its excessive degradation might result in the failure of immunosurveillance and hence promote immune escape. In this context, it appears plausible that ICD inducing chemotherapies can be improved in their efficacy by increasing autophagy (to stimulate ATP release from cancer cells), by selective inhibiting ectonucleotidase activity, or by A2AR receptor blockage. ${ }^{13,108,109}$ Whether such combination regimens might cause autoimmune reactions as an undesired side effect remains to be explored in detail. At present, an entire arsenal of autophagy inducers is available which might be considered for therapeutic intervention. Such autophagy inducers include mTORC1 inhibitors such as rapamycin and its analogues (rapalogs) everolimus and temsirolimus, as well as caloric restriction mimetics. ${ }^{61}$ In addition, small molecular inhibitors and antibodies targeting ectonucleotidases or adenosinergic receptors are under development. Future investigation will determine which among these agents will be the first to gain FDA approval.

\section{ACKNOWLEDGEMENTS}

$\mathrm{PL}$ is supported by the Chinese scholarship council and GK is supported by the Ligue contre le Cancer (équipe labelisée); Agence National de la Recherche (ANR) - Projets blancs; ANR under the frame of E-Rare-2, the ERA-Net for Research on Rare Diseases; Association pour la recherche sur le cancer (ARC); Cancéropôle lle-de-France; Institut National du Cancer (INCa); Institut Universitaire de France; Fondation pour la Recherche Médicale (FDM20140630126 and FDM 40739); the European Commission (ArtForce); the European Research Council (ERC); the LeDucq Foundation; the LabEx Immuno-Oncology; the RHU Torino Lumière, the SIRIC Stratified Oncology Cell DNA Repair and Tumor Immune Elimination (SOCRATE); the SIRIC Cancer Research and Personalized Medicine (CARPEM); and the Paris Alliance of Cancer Research Institutes (PACRI).

\section{CONFLICT OF INTEREST}

The authors declare no conflict of interest.

\section{REFERENCES}

1. Garrett RH, Grisham CM. Biochemistry. Belmont, CA, USA: Mary Finch; 2009.

2. Antonioli L, Pacher P, Vizi ES, Hasko G. CD39 and CD73 in immunity and inflammation. Trends Mol Med. 2013;19:355-367.

3. Forrester T, Williams CA. Release of adenosine triphosphate from isolated adult heart cells in response to hypoxia. J Physiol. 1977;268:371-390.

4. Burnstock G. Neural nomenclature. Nature. 1971;229:282-283.

5. Lazarowski ER, Boucher RC. Purinergic receptors in airway epithelia. Curr Opin Pharmacol. 2009;9:262-267.
6. Kroemer G, Galluzzi L, Kepp O, Zitvogel L. Immunogenic cell death in cancer therapy. Annu Rev Immunol. 2013;31:51-72.

7. Elliott MR, Chekeni FB, Trampont PC, et al. Nucleotides released by apoptotic cells act as a find-me signal to promote phagocytic clearance. Nature. 2009;461:282-286.

8. Gessi S, Merighi S, Sacchetto V, Simioni C, Borea PA. Adenosine receptors and cancer. Biochim Biophys Acta. 2011;1808:1400-1412.

9. Galluzzi L, Buque A, Kepp O, Zitvogel L, Kroemer G. Immunogenic cell death in cancer and infectious disease. Nat Rev Immunol. 2017;17:97-111.

10. Ghiringhelli F, Apetoh L, Tesniere A, et al. Activation of the NLRP3 inflammasome in dendritic cells induces IL-1beta-dependent adaptive immunity against tumors. Nat Med. 2009;15:1170-1178.

11. Deaglio S, Dwyer KM, Gao W, et al. Adenosine generation catalyzed by CD39 and CD73 expressed on regulatory T cells mediates immune suppression. J Exp Med. 2007;204:1257-1265.

12. Borsellino G, Kleinewietfeld M, Di Mitri D, et al. Expression of ectonucleotidase CD39 by Foxp3+ Treg cells: Hydrolysis of extracellular ATP and immune suppression. Blood. 2007;110:1225-1232.

13. Beavis PA, Milenkovski N, Henderson MA, et al. Adenosine receptor 2A blockade increases the efficacy of anti-PD-1 through enhanced antitumor t-cell responses. Cancer Immunol Res. 2015;3:506-517.

14. Young A, Ngiow SF, Barkauskas DS, et al. Co-inhibition of CD73 and A2AR adenosine signaling improves anti-tumor immune responses. Cancer Cell. 2016;30:391-403.

15. Fuchs $\mathrm{Y}$, Steller $\mathrm{H}$. Programmed cell death in animal development and disease. Cell. 2011;147:742-758.

16. Kroemer G, Dallaporta B, Resche-Rigon M. The mitochondrial death/life regulator in apoptosis and necrosis. Annu Rev Physiol. 1998;60:619-642.

17. Garg AD, Krysko DV, Verfaillie T, et al. A novel pathway combining calreticulin exposure and ATP secretion in immunogenic cancer cell death. EMBO J. 2012;31:1062-1079.

18. Fucikova J, Moserova I, Truxova I, et al. High hydrostatic pressure induces immunogenic cell death in human tumor cells. Int J Cancer. 2014:135:1165-1177.

19. Obeid M, Tesniere A, Ghiringhelli F, et al. Calreticulin exposure dictates the immunogenicity of cancer cell death. Nat Med. 2007;13:54-61.

20. Obeid M, Panaretakis T, Joza N, et al. Calreticulin exposure is required for the immunogenicity of gamma-irradiation and UVC lightinduced apoptosis. Cell Death Differ. 2007;14:1848-1850.

21. Zhou H, Forveille S, Sauvat A, et al. The oncolytic peptide LTX-315 triggers immunogenic cell death. Cell Death Dis. 2016;7:e2134.

22. Tesniere A, Schlemmer F, Boige V, et al. Immunogenic death of colon cancer cells treated with oxaliplatin. Oncogene. 2010;29:482-491.

23. Menger L, Vacchelli E, Adjemian S, et al. Cardiac glycosides exert anticancer effects by inducing immunogenic cell death. Sci Transl Med. 2012;4:143ra199.

24. Ma Y, Adjemian S, Mattarollo SR, et al. Anticancer chemotherapyinduced intratumoral recruitment and differentiation of antigenpresenting cells. Immunity. 2013;38:729-741.

25. Aranda F, Bloy N, Pesquet J, et al. Immune-dependent antineoplastic effects of cisplatin plus pyridoxine in non-small-cell lung cancer. Oncogene. 2015;34:3053-3062.

26. Yang $\mathrm{H}, \mathrm{Ma} \mathrm{Y}$, Chen $\mathrm{G}$, et al. Contribution of RIP3 and MLKL to immunogenic cell death signaling in cancer chemotherapy. Oncoimmunology. 2016;5:e1149673.

27. Broz P, Monack DM. Newly described pattern recognition receptors team up against intracellular pathogens. Nat Rev Immunol. 2013;13:551-565.

28. Janeway CA Jr. Pillars article: Approaching the asymptote? Evolution and revolution in immunology. Cold Spring Harb Symp Quant Biol. 1989;54: 1-13. J Immunol. 2013;191:4475-4487. 
29. Fuchs $\mathrm{Y}$, Steller H. Live to die another way: Modes of programmed cell death and the signals emanating from dying cells. Nat Rev Mol Cell Biol. 2015;16:329-344.

30. Yatim N, Cullen S, Albert ML. Dying cells actively regulate adaptive immune responses. Nat Rev Immunol. 2017;17:262-275.

31. Vacchelli E, Ma Y, Baracco EE, et al. Chemotherapy-induced antitumor immunity requires formyl peptide receptor 1 . Science. 2015;350:972-978.

32. Sistigu A, Yamazaki T, Vacchelli E, et al. Cancer cell-autonomous contribution of type I interferon signaling to the efficacy of chemotherapy. Nat Med. 2014;20:1301-1309.

33. Apetoh L, Ghiringhelli F, Tesniere A, et al. Toll-like receptor 4-dependent contribution of the immune system to anticancer chemotherapy and radiotherapy. Nat Med. 2007;13:1050-1059.

34. van Kempen TS, Wenink MH, Leijten EF, Radstake TR, Boes M. Perception of self: Distinguishing autoimmunity from autoinflammation. Nat Rev Rheumatol. 2015;11:483-492.

35. Cekic C, Linden J. Purinergic regulation of the immune system. Nat Rev Immunol. 2016;16:177-192.

36. Chekeni FB, Elliott MR, Sandilos JK, et al. Pannexin 1 channels mediate 'find-me' signal release and membrane permeability during apoptosis. Nature. 2010;467:863-867.

37. Boyd-Tressler A, Penuela S, Laird DW, Dubyak GR. Chemotherapeutic drugs induce ATP release via caspase-gated pannexin-1 channels and a caspase/pannexin-1-independent mechanism. J Biol Chem. 2014;289:27246-27263.

38. Lew MJ, White TD. Release of endogenous ATP during sympathetic nerve stimulation. Br J Pharmacol. 1987;92:349-355.

39. Stout CE, Costantin JL, Naus CC, Charles AC. Intercellular calcium signaling in astrocytes via ATP release through connexin hemichannels. J Biol Chem. 2002;277:10482-10488.

40. Bao L, Locovei S, Dahl G. Pannexin membrane channels are mechanosensitive conduits for ATP. FEBS Lett. 2004;572:65-68.

41. Lazarowski ER, Sesma JI, Seminario-Vidal L, Kreda SM. Molecular mechanisms of purine and pyrimidine nucleotide release. Adv Pharmacol. 2011;61:221-261.

42. Irvin JL, Irvin EM. The interaction of quinacrine with adenine nucleotides. J Biol Chem. 1954;210:45-56.

43. Bodin P, Burnstock G. Evidence that release of adenosine triphosphate from endothelial cells during increased shear stress is vesicular. J Cardiovasc Pharmacol. 2001;38:900-908.

44. Lazarowski ER. Vesicular and conductive mechanisms of nucleotide release. Purinergic Signal. 2012;8:359-373.

45. Chen $Y$, Yao $Y$, Sumi $Y$, et al. Purinergic signaling: A fundamental mechanism in neutrophil activation. Sci Signal. 2010;3:ra45.

46. Schwiebert EM, Egan ME, Hwang TH, et al. CFTR regulates outwardly rectifying chloride channels through an autocrine mechanism involving ATP. Cell. 1995;81:1063-1073.

47. Nichols CG. KATP channels as molecular sensors of cellular metabolism. Nature. 2006;440:470-476.

48. Abraham EH, Prat AG, Gerweck L, et al. The multidrug resistance (mdr1) gene product functions as an ATP channel. Proc Natl Acad Sci USA. 1993;90:312-316.

49. Das B, Mondragon MO, Sadeghian M, Hatcher VB, Norin AJ. A novel ligand in lymphocyte-mediated cytotoxicity: Expression of the beta subunit of $\mathrm{H}+$ transporting ATP synthase on the surface of tumor cell lines. J Exp Med. 1994;180:273-281.

50. Robertson J, Lang S, Lambert PA, Martin PE. Peptidoglycan derived from Staphylococcus epidermidis induces Connexin43 hemichannel activity with consequences on the innate immune response in endothelial cells. Biochem J. 2010;432:133-143.

51. Qu Y, Misaghi S, Newton K, et al. Pannexin-1 is required for ATP release during apoptosis but not for inflammasome activation. J Immunol. 2011;186:6553-6561.
52. Martins I, Wang Y, Michaud M, et al. Molecular mechanisms of ATP secretion during immunogenic cell death. Cell Death Differ. 2014;21:79-91.

53. Aymeric L, Apetoh L, Ghiringhelli F, et al. Tumor cell death and ATP release prime dendritic cells and efficient anticancer immunity. Cancer Res. 2010;70:855-858.

54. Martins I, Tesniere A, Kepp O, et al. Chemotherapy induces ATP release from tumor cells. Cell Cycle. 2009;8:3723-3728.

55. Pradelli LA, Villa E, Zunino B, Marchetti S, Ricci JE. Glucose metabolism is inhibited by caspases upon the induction of apoptosis. Cell Death Dis. 2014;5:e1406.

56. Kroemer G, Marino G, Levine B. Autophagy and the integrated stress response. Mol Cell. 2010;40:280-293.

57. Fader CM, Aguilera MO, Colombo MI. ATP is released from autophagic vesicles to the extracellular space in a VAMP7-dependent manner. Autophagy. 2012;8:1741-1756.

58. Michaud M, Martins I, Sukkurwala AQ, et al. Autophagy-dependent anticancer immune responses induced by chemotherapeutic agents in mice. Science. 2011;334:1573-1577.

59. Uhl M, Kepp O, Jusforgues-Saklani H, Vicencio JM, Kroemer G, Albert ML. Autophagy within the antigen donor cell facilitates efficient antigen cross-priming of virus-specific CD8+ T cells. Cell Death Differ. 2009;16:991-1005.

60. Pietrocola F, Pol J, Kroemer G. Fasting improves anticancer immunosurveillance via autophagy induction in malignant cells. Cell Cycle. 2016;15:3327-3328.

61. Pietrocola F, Pol J, Vacchelli E, et al. Caloric restriction mimetics enhance anticancer immunosurveillance. Cancer Cell. 2016;30:147-160.

62. Pulte D, Furman RR, Broekman MJ, et al. CD39 expression on T lymphocytes correlates with severity of disease in patients with chronic lymphocytic leukemia. Clin Lymphoma Myeloma Leuk. 2011;11:367-372.

63. Cai XY, Wang XF, Li J, et al. Overexpression of CD39 and high tumoral CD39(+)/CD8(+) ratio are associated with adverse prognosis in resectable gastric cancer. Int J Clin Exp Pathol. 2015;8:14757-14764.

64. Xu S, Shao QQ, Sun JT, et al. Synergy between the ectoenzymes CD39 and CD73 contributes to adenosinergic immunosuppression in human malignant gliomas. Neuro Oncol. 2013;15:1160-1172.

65. Aliagas E, Vidal A, Texido L, Ponce J, Condom E, Martin-Satue M. High expression of ecto-nucleotidases CD39 and CD73 in human endometrial tumors. Mediators Inflamm. 2014;2014:509027.

66. Kunzli BM, Bernlochner MI, Rath S, et al. Impact of CD39 and purinergic signalling on the growth and metastasis of colorectal cancer. Purinergic Signal. 2011;7:231-241.

67. Kunzli BM, Berberat PO, Giese T, et al. Upregulation of CD39/ NTPDases and P2 receptors in human pancreatic disease. Am J Physiol Gastrointest Liver Physiol. 2007;292:G223-G230.

68. Kansas GS, Wood GS, Tedder TF. Expression, distribution, and biochemistry of human CD39. Role in activation-associated homotypic adhesion of lymphocytes. J Immunol. 1991;146:2235-2244.

69. Ghiringhelli F, Bruchard M, Chalmin F, Rebe C. Production of adenosine by ectonucleotidases: A key factor in tumor immunoescape. J Biomed Biotechnol. 2012;2012:473712.

70. Chalmin F, Mignot G, Bruchard M, et al. Stat3 and Gfi-1 transcription factors control Th17 cell immunosuppressive activity via the regulation of ectonucleotidase expression. Immunity. 2012;36:362-373.

71. Sun $X$, Wu Y, Gao W, et al. CD39/ENTPD1 expression by CD4+Foxp3+ regulatory T cells promotes hepatic metastatic tumor growth in mice. Gastroenterology. 2010;139:1030-1040.

72. Rao S, Tortola L, Perlot T, et al. A dual role for autophagy in a murine model of lung cancer. Nat Commun. 2014; 5:3056.

73. Resta R, Yamashita Y, Thompson LF. Ecto-enzyme and signaling functions of lymphocyte CD73. Immunol Rev. 1998;161:95-109.

74. Loi S, Pommey S, Haibe-Kains B, et al. CD73 promotes anthracycline resistance and poor prognosis in triple negative breast cancer. Proc Natl Acad Sci USA. 2013;110:11091-11096. 
75. Turcotte M, Spring K, Pommey S, et al. CD73 is associated with poor prognosis in high-grade serous ovarian cancer. Cancer Res. 2015;75:4494-4503.

76. Yang Q, Du J, Zu L. Overexpression of CD73 in prostate cancer is associated with lymph node metastasis. Pathol Oncol Res. 2013;19:811-814.

77. Quezada C, Garrido W, Oyarzun C, et al. 5'-ectonucleotidase mediates multiple-drug resistance in glioblastoma multiforme cells. J Cell Physiol. 2013;228:602-608.

78. Coustan-Smith E, Song G, Clark C, et al. New markers for minimal residual disease detection in acute lymphoblastic leukemia. Blood. 2011;117:6267-6276.

79. Airas L, Jalkanen S. CD73 mediates adhesion of B cells to follicular dendritic cells. Blood. 1996;88:1755-1764.

80. Synnestvedt K, Furuta GT, Comerford KM, et al. Ecto-5'nucleotidase (CD73) regulation by hypoxia-inducible factor-1 mediates permeability changes in intestinal epithelia. J Clin Invest. 2002;110:993-1002.

81. Ledoux S, Runembert I, Koumanov K, Michel JB, Trugnan G, Friedlander G. Hypoxia enhances Ecto-5'-Nucleotidase activity and cell surface expression in endothelial cells: Role of membrane lipids. Circ Res. 2003;92:848-855.

82. Kalsi K, Lawson C, Dominguez M, Taylor P, Yacoub MH, Smolenski RT. Regulation of ecto-5'-nucleotidase by TNF-alpha in human endothelial cells. Mol Cell Biochem. 2002;232:113-119.

83. Stagg J, Beavis PA, Divisekera U, et al. CD73-deficient mice are resistant to carcinogenesis. Cancer Res. 2012;72:2190-2196.

84. Stagg J, Smyth MJ. Extracellular adenosine triphosphate and adenosine in cancer. Oncogene. 2010;29:5346-5358.

85. Lohman AW, Billaud M, Isakson BE. Mechanisms of ATP release and signalling in the blood vessel wall. Cardiovasc Res. 2012;95:269-280.

86. Jacobson KA, Muller CE. Medicinal chemistry of adenosine, P2Y and P2X receptors. Neuropharmacology. 2016;104:31-49.

87. Di Virgilio F, Adinolfi E. Extracellular purines, purinergic receptors and tumor growth. Oncogene. 2017;36:293-303.

88. Hill LM, Gavala ML, Lenertz LY, Bertics PJ. Extracellular ATP may contribute to tissue repair by rapidly stimulating purinergic receptor $X 7$ dependent vascular endothelial growth factor release from primary human monocytes. J Immunol. 2010;185:3028-3034.

89. Furman D, Chang J, Lartigue L, et al. Expression of specific inflammasome gene modules stratifies older individuals into two extreme clinical and immunological states. Nat Med. 2017;23:174-184.

90. Ma Y, Aymeric L, Locher C, et al. Contribution of IL-17-producing gamma delta $\mathrm{T}$ cells to the efficacy of anticancer chemotherapy. J Exp Med. 2011;208:491-503.

91. Roger S, Jelassi B, Couillin I, Pelegrin P, Besson P, Jiang LH. Understanding the roles of the P2X7 receptor in solid tumour progression and therapeutic perspectives. Biochim Biophys Acta. 2015;1848:2584-2602.

92. Zhang X, Qin J, Zou J, et al. Extracellular ADP facilitates monocyte recruitment in bacterial infection via ERK signaling. Cell Mol Immunol. 2016. [Epub ahead of print]. https://doi.org/10.1038/cmi.2016.56

93. Sitkovsky MV. T regulatory cells: Hypoxia-adenosinergic suppression and re-direction of the immune response. Trends Immunol. 2009;30:102-108.
94. Young A, Mittal D, Stannard K, et al. Co-blockade of immune checkpoints and adenosine A2A receptor suppresses metastasis. Oncoimmunology. 2014;3:e958952.

95. Wang L, Fan J, Thompson LF, et al. CD73 has distinct roles in nonhematopoietic and hematopoietic cells to promote tumor growth in mice. J Clin Invest. 2011;121:2371-2382.

96. Ohta A, Gorelik E, Prasad SJ, et al. A2A adenosine receptor protects tumors from antitumor T cells. Proc Natl Acad Sci USA. 2006;103:13132-13137.

97. Cekic C, Sag D, Li Y, Theodorescu D, Strieter RM, Linden J. Adenosine A2B receptor blockade slows growth of bladder and breast tumors. J Immunol. 2012;188:198-205.

98. Whiteside TL. Targeting adenosine in cancer immunotherapy: A review of recent progress. Expert Rev Anticancer Ther. 2017;17: 527-535.

99. Adams JL, Smothers J, Srinivasan R, Hoos A. Big opportunities for small molecules in immuno-oncology. Nat Rev Drug Discov. 2015;14:603-622.

100. Ohta A, Sitkovsky M. Role of G-protein-coupled adenosine receptors in downregulation of inflammation and protection from tissue damage. Nature. 2001;414:916-920.

101. Casares N, Pequignot MO, Tesniere A, et al. Caspase-dependent immunogenicity of doxorubicin-induced tumor cell death. J Exp Med. 2005;202:1691-1701.

102. Sukkurwala AQ, Adjemian S, Senovilla L, et al. Screening of novel immunogenic cell death inducers within the $\mathrm{NCI}$ Mechanistic Diversity Set. Oncoimmunology. 2014;3:e28473.

103. Pardoll DM. The blockade of immune checkpoints in cancer immunotherapy. Nat Rev Cancer. 2012;12:252-264.

104. Brahmer J, Reckamp KL, Baas P, et al. Nivolumab versus docetaxel in advanced squamous-cell non-small-cell lung cancer. N Engl J Med. 2015;373:123-135.

105. Chen DS, Mellman I. Elements of cancer immunity and the cancerimmune set point. Nature. 2017;541:321-330.

106. Teng MW, Ngiow SF, Ribas A, Smyth MJ. Classifying cancers based on T-cell infiltration and PD-L1. Cancer Res. 2015;75:2139-2145.

107. Pfirschke C, Engblom C, Rickelt S, et al. Immunogenic chemotherapy sensitizes tumors to checkpoint blockade therapy. Immunity. 2016;44:343-354.

108. Allard B, Pommey S, Smyth MJ, Stagg J. Targeting CD73 enhances the antitumor activity of anti-PD-1 and anti-CTLA-4 mAbs. Clin Cancer Res. 2013;19:5626-5635.

109. lannone R, Miele L, Maiolino P, Pinto A, Morello S. Adenosine limits the therapeutic effectiveness of anti-CTLA4 mAb in a mouse melanoma model. Am J Cancer Res. 2014;4:172-181. 